\title{
Knockout of Zn Transporters Zip-1 and Zip-3 Attenuates Seizure-Induced CA1 Neurodegeneration
}

\author{
Jing Qian, ${ }^{1}$ Kaiping Xu, ${ }^{1}$ Jong Yoo, ${ }^{1}$ Tim T. Chen, ${ }^{1}$ Glen Andrews, ${ }^{4}$ and Jeffrey L. Noebels $\mathbf{s}^{1,2,3}$ \\ Departments of ${ }^{1}$ Neurology, ${ }^{2}$ Neuroscience, and ${ }^{3}$ Molecular and Human Genetics, Baylor College of Medicine, Houston, Texas 77030, and ${ }^{4}$ Department of \\ Biochemistry and Molecular Biology, University of Kansas Medical Center, Kansas City, Kansas 66160
}

CA1 pyramidal neurons are the final integrators of information flow leaving the hippocampus, yet are singularly vulnerable to activitydependent cell death. Zinc (Zn) entry into cells may add to this vulnerability. Here, we find that Slc39a1 and Slc39a3, members of the Zip (Zrt/Irt-like protein) plasmalemmal Zn transporter family, are predominantly expressed in the hippocampus. We examined Zip-1,3deficient mice to investigate their role in neurodegeneration following intense synaptic activation. When isolated by blockade of NMDA receptors and voltage-gated calcium channels, the absence of both transporters slowed passive $\mathrm{Zn}$ uptake into CA1 neurons measured with intracellular fluorescent $\mathrm{Zn}$ dyes. In vivo $\mathrm{CA} 1$ cell damage following kainic acid exposure was greatly attenuated. Consistent with the hypothesis that $\mathrm{Zn}$ entry contributes to neurodegeneration, Znt-3-deficient mice lacking synaptic $\mathrm{Zn}$ also show less hippocampal cell damage following kainic acid injection. Zip transporters may provide selective therapeutic targets to protect these neurons from early Zn-induced neurodegeneration following injury.

\section{Introduction}

Hippocampal pyramidal neurons play key roles in learning and memory, yet are especially vulnerable to brain injury. Early neurodegeneration following seizures and ischemia is largely a consequence of glutamate excitotoxicity that activates NMDA receptors and Ca-permeable AMPA/kainate receptors, leading to elevated intracellular $\mathrm{Ca}^{2+}$ and eventual cell death (Kwak and Weiss, 2006; Liu and Zukin, 2007). However, attempts to block these entry routes have been therapeutically ineffective (Villmann and Becker, 2007; Besancon et al., 2008). Excessive zinc ( $\mathrm{Zn}$ ) translocation has also been proposed as a molecular trigger of the cellular apoptotic cascade (Weiss et al., 2000; Frederickson et al., 2005; Kwak and Weiss, 2006; Hershfinkel et al., 2009). Several possible routes of transmembrane $\mathrm{Zn}$ entry have been identified. When externally applied, Zn permeates cultured neurons through NMDA receptors, Ca-permeable AMPA/Kainate receptors, voltage-gated calcium channels, and neuronal plasma membrane Zn transporters (Freund and Reddig, 1994; Sensi et al., 1997, 1999; Colvin et al., 2003). Of the latter, members of the solute carrier 39a (Slc39a) Zn transporter family called Zips (Zrt/Irt-like protein) are interesting candidates, since they mediate inward $\mathrm{Zn}$ transport and are expressed in brain (Dufner-Beattie et al., 2003, Belloni-Olivi et al., 2009, Emmetsberger et al., 2010).

The contribution of $\mathrm{Zn}$ accumulation to neurodegeneration following brain injury has been extensively explored. Chelation

\footnotetext{
Received 0ct. 1, 2010; accepted 0ct. 20, 2010.

This work was supported by National Institute of Neurological Disorders and Stroke Grant NS 29709 (J.L.N.) and the Blue Bird Circle Pediatric Neurology Research Foundation. We are grateful to Ed Glasscock for generous assistance.

Address correspondence to Dr. Jeffrey L. Noebels, Department of Neurology, Baylor College of Medicine, Houston, TX 77030.E-mail: jnoebels@bcm.tmc.edu.

DOI:10.1523/JNEUROSCI.5162-10.2011

Copyright $\odot 2011$ the authors $\quad 0270-6474 / 11 / 310097-08 \$ 15.00 / 0$
}

of extracellular $\mathrm{Zn}$ is the most widely used technique. While in some studies, Zn chelation successfully protects neurons (Koh et al., 1996), in others Zn removal increases cell death (Domínguez et al., 2003; Côté et al., 2005; Lavoie et al., 2007). Lowering brain $\mathrm{Zn}$ content by a low $\mathrm{Zn}$ diet or genetic ablation of presynaptic vesicular $\mathrm{Zn}$ transporters also fails to prevent neurodegeneration (Lee at al, 2000; Takeda et al., 2005). In these studies, however, it is unclear to what extent the reduction of neuronal $\mathrm{Zn}$ pools actually affected postsynaptic Zn entry. Since extracellular Zn can modulate membrane excitability by targeting receptors for NMDA, GABA $A$, glycine, voltage-gated $\mathrm{Ca}^{2+}$ channels, and ATP-K ${ }^{+}$channels (Westbrook and Mayer, 1987; Bancila et al., 2004; Hirzel et al., 2006; Cataldi et al., 2007; Traboulsie et al., 2007), any experimental removal of $\mathrm{Zn}$ (by dietary deficiency, by reduction in the quantal size of presynaptic vesicular $\mathrm{Zn}$ pools, or particularly by chelation) before it enters postsynaptic neurons may mask the actual amount of $\mathrm{Zn}$ movement into cells. For example, endogenous $\mathrm{Zn}$ inhibits NMDA receptors (Vogt et al., 2000; Molnár and Nadler, 2001), but chelation of extracellular Zn greatly magnifies the effects of NMDA receptor excitotoxicity (Domínguez et al., 2006), allowing more Ca entry, thereby obscuring the ionic mechanisms contributing to cell death.

To isolate the contribution of extracellular Zn entry to neurodegeneration following brain injury while sparing other receptors and channels essential for hippocampal signaling, a means of directly reducing $\mathrm{Zn}$ entry into postsynaptic neurons without perturbing upstream $\mathrm{Zn}$ availability is required. In the present study, we combined fluorescent $\mathrm{Zn}$ dye imaging, electrophysiology, and histochemistry methods to demonstrate that two members of the plasma membrane inward $\mathrm{Zn}$ transporter family, Slc39a1 (Zip-1) and Slc39a3 (Zip-3) are strongly expressed in hippocampal pyramidal neurons and contribute to $\mathrm{Zn}$ homeostasis. When normalized for the amount of electrographic 
seizure activity within a $24 \mathrm{~h}$ period following kainate injection, we found a significant reduction of cell damage in CA1 principal cells in mice lacking both $Z i p-1$ and Zip-3 transporters compared with wild-type littermates. Our results identify Zip-1,3 transporters as novel therapeutic targets to protect CA1 neurons from $\mathrm{Zn}$ entry-induced neuronal degeneration following seizure or ischemia attacks.

\section{Materials and Methods}

Generation of Zip-1 and Zip-3 double-knockout mice. The targeted disruption of both Zip-1 and $\mathrm{Zip}$-3 gene expression in mouse has been previously described in detail by DufnerBeattie et al. (2006). Briefly, the targeting construct fused the initiator methionine codon of the targeted Zip gene with the open reading frame of the enhanced green fluorescent protein (EGFP) reporter. This disrupts the protein coding sequence of the targeted $\mathrm{Zip}$ gene and inserts a stop codon and a polyadenylation signal into exon 2 of the gene. This allows for EGFP expression that is driven by the targeted

Zip promoter and disruption of the targeted Zip gene expression. The loxP flanked MC1-Neo cassette was removed from the knock-out insertion in targeted Zip-1 and Zip-3 mice by mating with Cre-expressing transgenic mice (Jackson Laboratories). Both strains of knock-out mice were generated by inbreeding the neo-negative Zip-1 or 3-/- offspring from the breeding with Cre-expressing transgenic mice. Homozygous (Zip-1 -/- and Zip-3-/-) mice were crossbred, and then offspring were inbred to generate homozygous $Z i p-1, Z i p-3$ double-knock-out mice.

Whole-cell patching and optical recording. Hippocampal slices were prepared from Zip-1,3 double-knock-out mice and homozygous wild-type littermates of the same strain using a vibratome (350 $\mu \mathrm{m}$ thickness) as described in a previous study (Qian and Noebels, 2005). Brain slices were incubated in artificial CSF (ACSF) at $32^{\circ} \mathrm{C}$ for $1 \mathrm{~h}$ and then transferred into a submerged recording chamber mounted on a microscope (Axioskop 2, Zeiss) for dye loading and electrophysiology recording. Brain slices were constantly perfused with ACSF contained the following (in mM): $125 \mathrm{NaCl}$, $2.5 \mathrm{KCl}, 2.5 \mathrm{CaCl}_{2}, 25 \mathrm{NaHCO}_{3}, 2 \mathrm{MgSO}_{4}, 1.25 \mathrm{NaH}_{2} \mathrm{PO}_{4}$, and 11 D-glucose, gassed with $95 \% \mathrm{O}_{2} / 5 \% \mathrm{CO}_{2}$ to maintain a constant $\mathrm{pH}$ of 7.4. The temperature of the recording chamber was controlled at $30^{\circ} \mathrm{C}$. The microscope was fitted with a $40 \times / 0.8$ numerical aperture Zeiss water-immersion objective and differential interference contrast optics to visualize the hippocampal slices. CA1 pyramidal neurons were identified based on their location in the stratum pyramidale and characteristic morphology (pyramidal shape of the cell body and the distinct apical dendritic trunk). Whole-cell recording pipettes (4-6 M $\Omega$ ) were pulled from borosilicate glass and filled with the following (in mM): 107.5 Cs-gluconate, $10 \mathrm{KCl}, 20 \mathrm{HEPES}, 8 \mathrm{NaCl}, 6$ phosphocreatine, 5 QX-314, $4 \mathrm{Mg}$-ATP, $0.3 \mathrm{Na}-\mathrm{GTP}$, and 0.2 EGTA, pH 7.3. The membrane-impermeable fluorescent dyes Alexa $586(4 \mu \mathrm{M})$ and FluoZin-3 $(40 \mu \mathrm{M})$ were added to the pipette solution. The superficial layer of cells damaged during cutting was carefully removed over the CA1 area by gentle suction through a polished pipette, and whole-cell patchclamp recordings were then made from the visually identified CA1 pyramidal somata with an Molecular Devices Axoclamp-1D amplifier. The membrane potential was clamped at $-70 \mathrm{mV}$. The measured input membrane resistance was in the range of $60-100 \mathrm{M} \Omega$. Optical recordings were made $30 \mathrm{~min}$ after membrane break-in. To assay passive $\mathrm{Zn}$ uptake, $\mathrm{NaH}_{2} \mathrm{PO}_{4}$ was removed from the ACSF solution during perfusion of 10 $\mu \mathrm{M} \mathrm{Zn}{ }^{2+}$ to avoid precipitation of $\mathrm{Zn}$ (Frederickson et al., 2006), which may reduce the actual $\mathrm{Zn}^{2+}$ concentration applied.

Fluorescence images were obtained with a NeuroCCD-SMQ $(80 \times 80)$ imaging system (RedshirtImaging). For Alexa 586, the excitation filter was $580 / 20 \mathrm{~nm}$ and the emission filter was $630 / 60 \mathrm{~nm}$. For FluoZin-3, the excitation filter was $488 / 20 \mathrm{~nm}$ and the emission filter was 535/25 nm. The GFP image was obtained using the same optical setting as for FluoZin-3. Each sample consisted of paired Alexa 586 and FluoZin-3 fluorescence images. Autofluorescence was corrected by subtracting the fluorescence intensity measured from an area within $50 \mu \mathrm{m}$ devoid of neuronal processes of the dye-loaded cell. The frame rate of the CCD camera was set at $40 \mathrm{~Hz}$ and an average of 50 frames per image was used for data analysis.

Kainic acid injection and detection of cell injury. Adult mice from mutant and wild-type genotypes (30-35 d of age) were injected intraperitoneally with kainic acid (KA) (either $15 \mathrm{mg} / \mathrm{kg}$ or a higher dose of 30 $\mathrm{mg} / \mathrm{kg}$ consisting of two injections of $15 \mathrm{mg} / \mathrm{kg}$ separated by $1 \mathrm{~h}$ ). Early neurodegeneration following KA injection was assayed by the FluoroJade (FJ) staining technique. Briefly, $24 \mathrm{~h}$ after the KA injection, mice were killed, and the brains were quickly removed and placed into PBS containing $4 \%$ paraformaldehyde at $4^{\circ} \mathrm{C}$ for overnight fixation. Brains were then infiltrated with a sucrose tissue-freezing solution and then frozen at $-80^{\circ} \mathrm{C}$. Transverse sections $(20 \mu \mathrm{m}$ thick) were cut in a cryostat from the midsection of hippocampi (the same portion of hippocampi as used in electrophysiology experiments) and mounted onto glass coverslips for Fluoro-Jade staining. The staining procedures were followed according to the instructions for the Fluoro-Jade detection kit (Millipore Bioscience Research Reagents).

The areal intensity of Fluoro-Jade staining in the hippocampal formation was measured by calculating an FJ staining index (FJI) for each section, determined by the number of Fluoro-Jade-positive pixels in the pyramidal cell layer region of interest (CA1, CA3). This value was normalized by the length of the whole pyramidal cell layer in each section, and compared across genotypes.

Video/electroencephalographic recording. Mice ranging from 30 to $35 \mathrm{~d}$ of age were surgically implanted under avertin anesthesia with a microminiature connector attached to silver wire electrodes ( 0.005 inch diameter). EEG electrodes were positioned through cranial burr holes overlying the cortical surface in the subdural space over the brain. After $\geq 1$ d of postsurgical recovery, the cortical EEG activity of freely moving mice was monitored before and after intraperitoneal KA injection. Electrographic seizure activity was visually identified and quantified by measuring the time of onset and the total aggregate time spent in synchronized epileptiform EEG discharge activity. A computer program was developed to plot the cumulative EEG discharge data as shown in Figure 4. The synchronized EEG discharges were classified into two categories: typical seizure-like synchronized EEG discharges that exhibit a burst frequency of $>1 \mathrm{~Hz}$; and discharges with a burst frequency $<1 \mathrm{~Hz}$. 
A
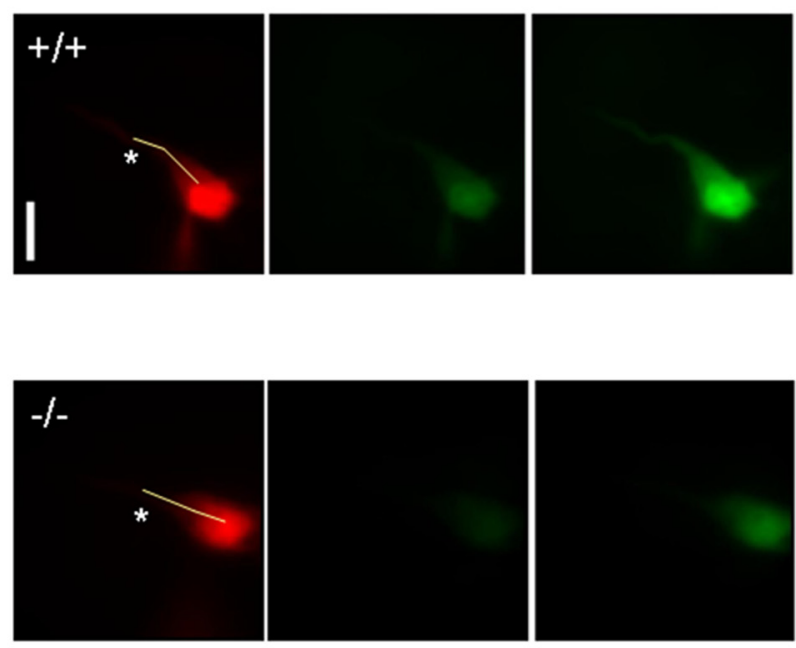

B
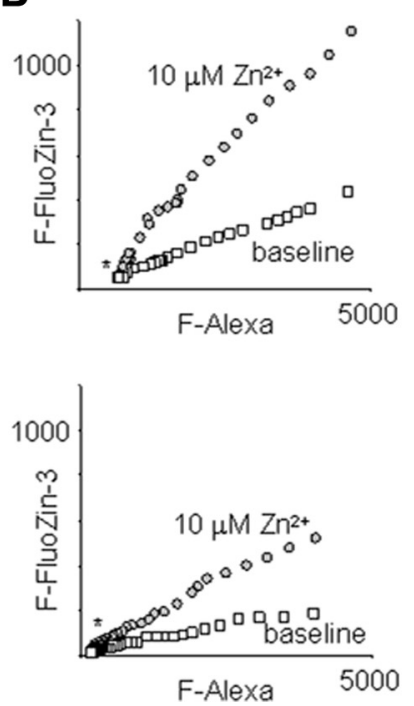

C
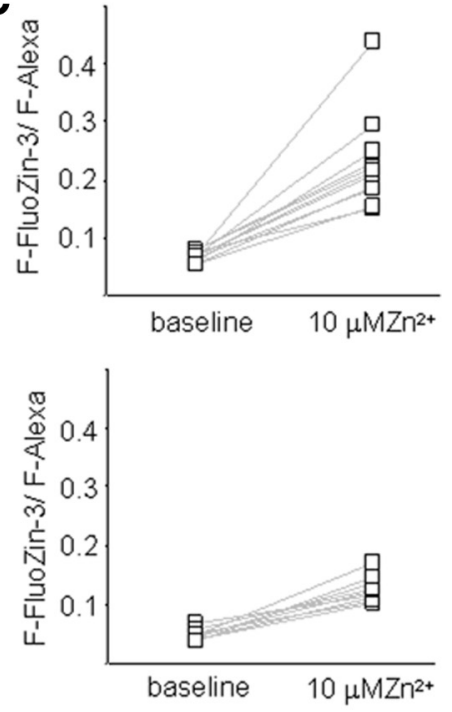

Figure 2. Slowed Zn uptake in mice lacking Zip-1,3 transporters. A, Fluorescence images taken from a representative pyramidal neuron filled with both Alexa and the Zn dye FluoZin-3 in wild-type (top) and mutant (bottom) neurons. Top left, Alexa image. scale bar, $10 \mu \mathrm{m}$. Top center, FluoZin-3 image taken in control solution. Top right, FluoZin-3 image taken after the exposure of $10 \mu \mathrm{m}$ $\mathrm{Zn}^{2+}$ to slice for $30 \mathrm{~min}$. $\boldsymbol{B}$, Linear relationship between FluoZin-3 fluorescence (F-FluoZin-3) and Alexa fluorescence (F-Alexa) sampled across the cell soma/proximal dendrite surface in the sample cell as marked by the line in the Alexa image in $\boldsymbol{A}$. The F-FluoZin-3/F-Alexa ratio is quite homogeneous across the cell surface. Therefore, the ratio of F-FluoZin-3/F-Alexa before and after $\mathrm{Zn}{ }^{2+}$ exposure was measured to minimize the sampling variance in $Z n$ uptake. C, Summary data for passive $Z n$ uptake assay for $+/+$ and $-/-\mathrm{groups}$. A 30 min perfusion of $10 \mu \mathrm{m} Z \mathrm{n}^{2+}$ increases the ratio of F-FluoZin-3/F-Alexa from $6.7 \pm 0.9 \%$ to $23.1 \pm 8.1 \%(n=11)$. The corresponding result for the $-/-$ group is $4.8 \pm 0.9 \%$ and $12.1 \pm 2.2 \%$ ( $n=9)$, a $\sim 50 \%$ reduction $(p<0.01)$, indicating that lack of Zip-1,3 Zn transporters in CA1 pyramidal neurons reduces Zn uptake in response to elevation of extracellular Zn concentration.

Western immunoblotting. Cortex and hippocampi were dissected from freshly isolated whole brain of $\mathrm{Zip}+/+$ and $-/-$ mice, flash frozen in liquid nitrogen, and subsequently homogenized on ice with a Tissue Tearor in lysis buffer containing the following (in mM): 20 Tris, $\mathrm{pH} 7.5$, $138 \mathrm{NaCl}, 3 \mathrm{KCl}, 1 \%$ Triton X-100, 1 EGTA, 2 EDTA, 1 benzamidine, 1 phenylmethylsulfonylfluoride, 1 dithiothreitol, and $5 \mu \mathrm{g} / \mathrm{ml}$ each of aprotinin, leupeptin, and pepstatin A. Total protein concentration of the brain and heart tissue lysates were determined using the Bio-Rad Protein Assay. One hundred micrograms of cortex or hippocampal protein lysate were separated on $8 \%$ Tris-HEPES-SDS polyacrylamide gels, analyzed by Western immunoblot using rabbit polyclonal anti-NMDA receptor 1 (NMDAR1) antibody (1:300 in vehicle; catalog \#4204, Cell Signaling Technology) and HRP-tagged goat anti-rabbit IgG secondary antibody (1:5000 dilution in vehicle, Santa Cruz Biotechnology), and subsequently detected using a commercial chemiluminescent substrate (SuperSignal, Pierce Chemical).

Data analysis. Data from mice in each genotype were pooled and represented with mean/SDs. The Student's $t$ test was used for significance analysis.

\section{Results}

\section{Expression of Zn transporter subfamily II members Zip-1} and $Z i p-3$ in the hippocampus

$Z i p-1$ - and Zip-3-containing neurons were visualized in the CNS by detecting the expression of an EGFP reporter engineered into mice doubly homozygous for targeted deletions of these genes (Dufner-Beattie et al., 2006). These transporters are expressed at low levels throughout the forebrain, brainstem, and cerebellum; however, they show a predominant localization within the hippocampal formation. Figure $1 \mathrm{~A}$ shows the intense EGFP signal found within the hippocampal formation after immunostaining with anti-GFP-Alexa 594 (Texas Red) in a coronal section from Zip-1,3 double-knock-out mouse brain. In comparison, antiGFP-Alexa 594 staining in $+/+$ brain shows no specific signal such as a highlighted staining in the pyramidal cell layers, as observed in the $-1-$ mouse brain (data not shown). The fluorescence intensity of EGFP antibody staining in the pyramidal cell layer is $\sim 50 \%$ higher than that in the nearby entorhinal cortex after being normalized by the cell density in each region (obtained from densitometry of adjacent Nissl-stained sections). Figure $1 B, C$ demonstrates that the Zip- 1 and Zip-3 expression in the hippocampal pyramidal cell layer arises from staining of the principal cells. To verify that the strong expression within the cell layer represented intrinsic transporter expression in individual pyramidal cells, a patch pipette was used to load visually identified CA1 pyramidal cells in Zip-1,3 mutant (Fig. $1 B$ ) and wildtype (Fig. 1C) mouse brain slices with the fluorescent tracer Alexa 568. In Zip-1,3-null mutants, but not in control wild-type mice, significant GFP fluorescence could be detected in pyramidal cells as shown in the corresponding EGFP image in Figure $1 B$. EGPF staining was also detected in cells at very low intensity similar to neocortex in other regions of brain including, thalamus, pons, and cerebellum (data not shown).

\section{Ablation of Zip-1 and Zip-3 transporters slows Zn uptake into CA1 pyramidal cells}

We used whole-cell patch-clamp recording combined with fluorescent imaging to examine the potential contribution of $Z i p-1,3$ transporters to $\mathrm{Zn}$ uptake into CA1 pyramidal neurons in vitro. We isolated the inward transporter-mediated fraction by adding the NMDA receptor antagonist D-APV and the AMPA receptor antagonist DNQX to the perfusate of hippocampal slices to eliminate possible $\mathrm{Zn}$ entry through glutamatergic receptors (GluRs). To prevent $\mathrm{Zn}$ entry through open voltage-gated $\mathrm{Ca}^{2+}$ channels in the target cell, the intracellular solution contained $5 \mathrm{~mm} \mathrm{QX-}$ 314 to block voltage-gated $\mathrm{Na}^{+}$channels, and the somatic membrane potential was constantly held at $-70 \mathrm{mV}$. To monitor intracellular Zn levels, single CA1 pyramidal neurons were loaded with the membrane-impermeable fluorescent tracer $\mathrm{Al}$ exa 568 and the Zn-sensitive dye FluoZin-3. A pair of Alexa 568 and FluoZin-3 fluorescence images, providing a baseline reference of intracellular $\mathrm{Zn}$ activity, was obtained $30 \mathrm{~min}$ after mem- 
brane break-in. We determined that the ratio F-FluoZin-3/F-Alexa is homogeneous across the cell surface from soma to the proximal one third of the apical dendrite, as demonstrated by a linear relationship between FluoZin-3 fluorescence (F-FluoZin-3) and Alexa fluorescence (FAlexa) sampled along the line shown in the Alexa image (Fig. $2 \mathrm{~A}$, left), indicating that cytoplasmic free $\mathrm{Zn}$ levels are buffered to a uniform level. To minimize the sampling variance, we measured the ratio F-FluoZin-3/F-Alexa, instead of F-FluoZin-3 alone, before (Fig. $2 A$, center) and after (Fig. 2A, right) exposure of the slice to extracellular $\mathrm{Zn}^{2+}$ to calculate $\mathrm{Zn}$ uptake. In wild-type brain slices, perfusion of 10 $\mu \mathrm{M} \mathrm{Zn}^{2+}$ for $30 \mathrm{~min}$ increased the F-FluoZin-3/F-Alexa ratio from $6.7 \pm 0.9 \%$ to $23.1 \pm 8.1 \%(n=11)$ (Fig. $2 A-C$, top). In Figure $2 A-C$ (bottom), the corresponding result for neurons in $Z i p-1 / 3-/-$ slices was $4.8 \pm 0.9 \%$ and $12.1 \pm 2.2 \%(n=9)$, which represents a significant reduction of $\sim 50 \%$ $(p<0.01)$ compared with wild-type neurons. This sharp reduction is consistent with the transporter localization obtained with EGFP staining (Fig. 1) and supports the notion that Zip-1,3 transporters expressed in hippocampal CA1 pyramidal cells regulate passive $\mathrm{Zn}$ homeostasis.

\section{Protection from seizure-induced cell injury in the CA1 area of Zip-1,3-/- mutants}

To determine whether this twofold reduction in the contribution of transportermediated Zn entry during intense synaptic activation could influence cell survival following injury in vivo, we used a widely used hippocampal convulsant that generates extensive excitotoxic pyramidal cell damage while allowing survival of the animal. Zip-1,3 mutants and wild-type littermates were injected intraperitoneally with KA (15 mg/kg), monitored by video EEG to quantify seizure activity, and early hippocampal cell injury was evaluated $24 \mathrm{~h}$ later with FJ staining. Figure $3 \mathrm{~A}$ shows representative images of FJ staining in the hippocampus of Zip$1 / 3-/-$ and $+/+$ brains analyzed $1 \mathrm{~d}$ after KA injection. The Fluoro-Jade staining is largely confined to the pyramidal cell layers except for the CA2 area. The dentate granule layer was not stained at all. This pattern of Fluoro-Jade staining is consistent with the cell death pattern in the hippocampus following excitotoxic insults.

In a large sample of mice from both genotypes, disruption of $Z i p-1,3$ transporters produced clear protection in the hippocampal CA1 region. We found positive cellular FJ staining in 21 of 41 injected $+/+$ mice in the CA1 area; in contrast, 11 of 43 injected $-/-$ mutants showed positive FJ staining in the same area. We calculated an FJI for hippocampal pyramidal subregions in each section to quantify cell injury in the mutant and littermate control genotypes. We found evidence that the two hippocampal
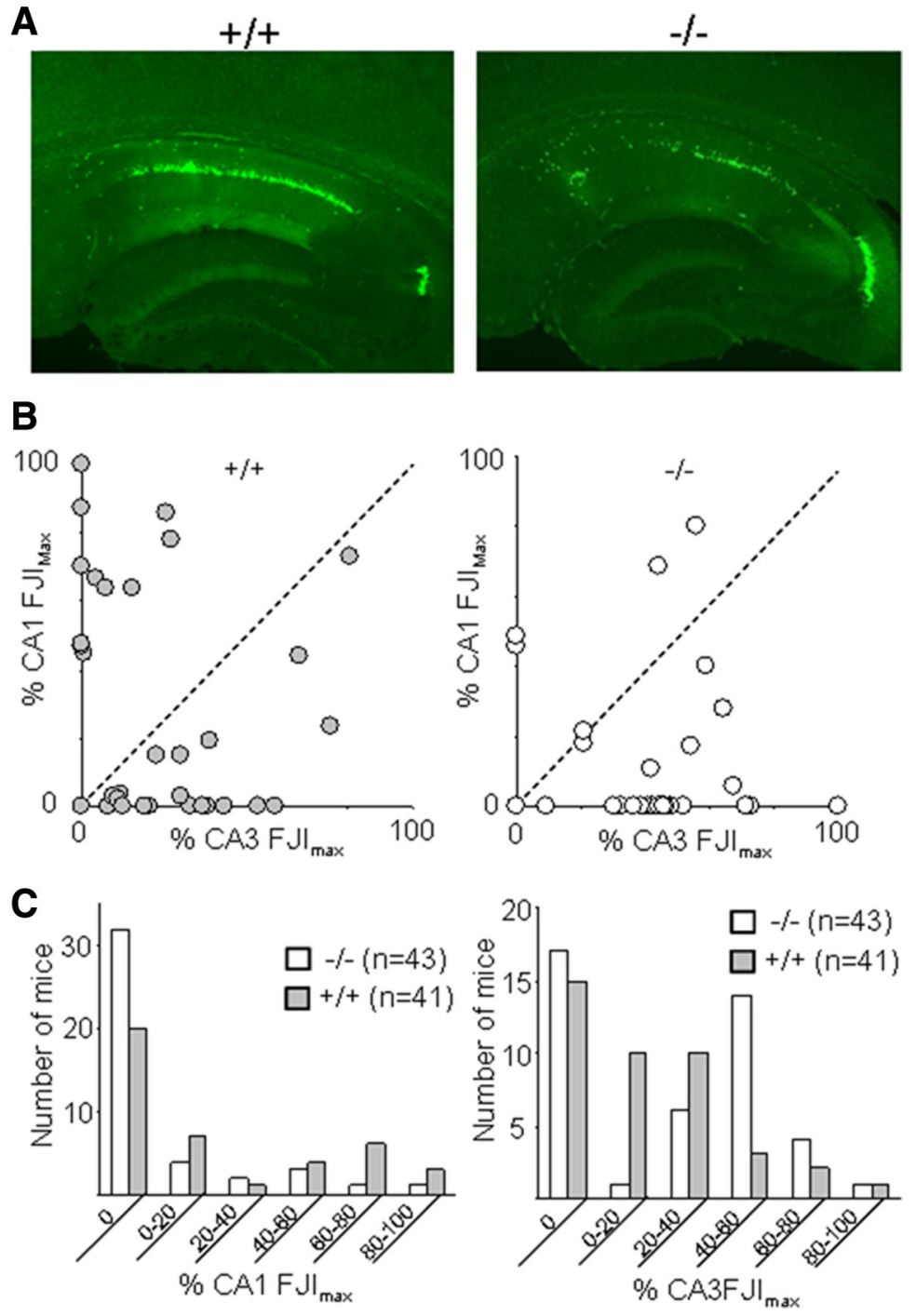

Figure 3. Attenuated neurodegeneration in the hippocampal CA1 area in Zip-1,3-/ - mutants after KA-induced seizures. $\boldsymbol{A}$, Representative Fluoro-Jade-stained sections taken from transverse mid-hippocampal sections from $+/+$ and $-/-$ mutant 作 (he upper left quadrant represents mice with selective and extensive CA1 cell injury but little CA3. This pattern is strikingly reduced in Zip-1,3-/- mutant mice (right). C, Summary data for the extent of CA1 and CA3 neurodegeneration $24 \mathrm{~h}$ after administration of KA (15 mg/kg).

subregions exhibit different vulnerability profiles to kainateinduced injury. The average FJI in CA1 for the $-/-$ group was $9 \pm 19 \%(n=43)$ of its maximal value, which is significantly smaller than the value for the $+/+$ group $(23 \pm 32 \%, n=41, p=$ $0.01)$. Only 5 of $43 \mathrm{Zip}-1,3-/-$ mice fell in the upper 60 percentile of FJIs compared with 13 of 41 from the $+/+$ group. These data demonstrate a striking reduction in early CA1 injury in the -I- group after KA injection compared with wild-type mice. The pattern of selective protection of CA1 neurons in $-/-$ mice was even more evident when cell damage from both subregions is directly compared, as shown in Figure $3 B$. In wild-type mice, a group of mice (8/41) exhibits a characteristic pattern of vulnerability exclusively confined to the CA1 pyramidal cell region (Fig. $3 B$, left). These mice display extensive CA1 FJ staining ( $>40 \%$ of maximum FJI), while the CA3 region is mostly spared $(<10 \%$ of maximum FJI). In the $-/-$ group (Fig. $3 B$, right), the number of mice with exclusive CA1 cell damage was greatly diminished. 

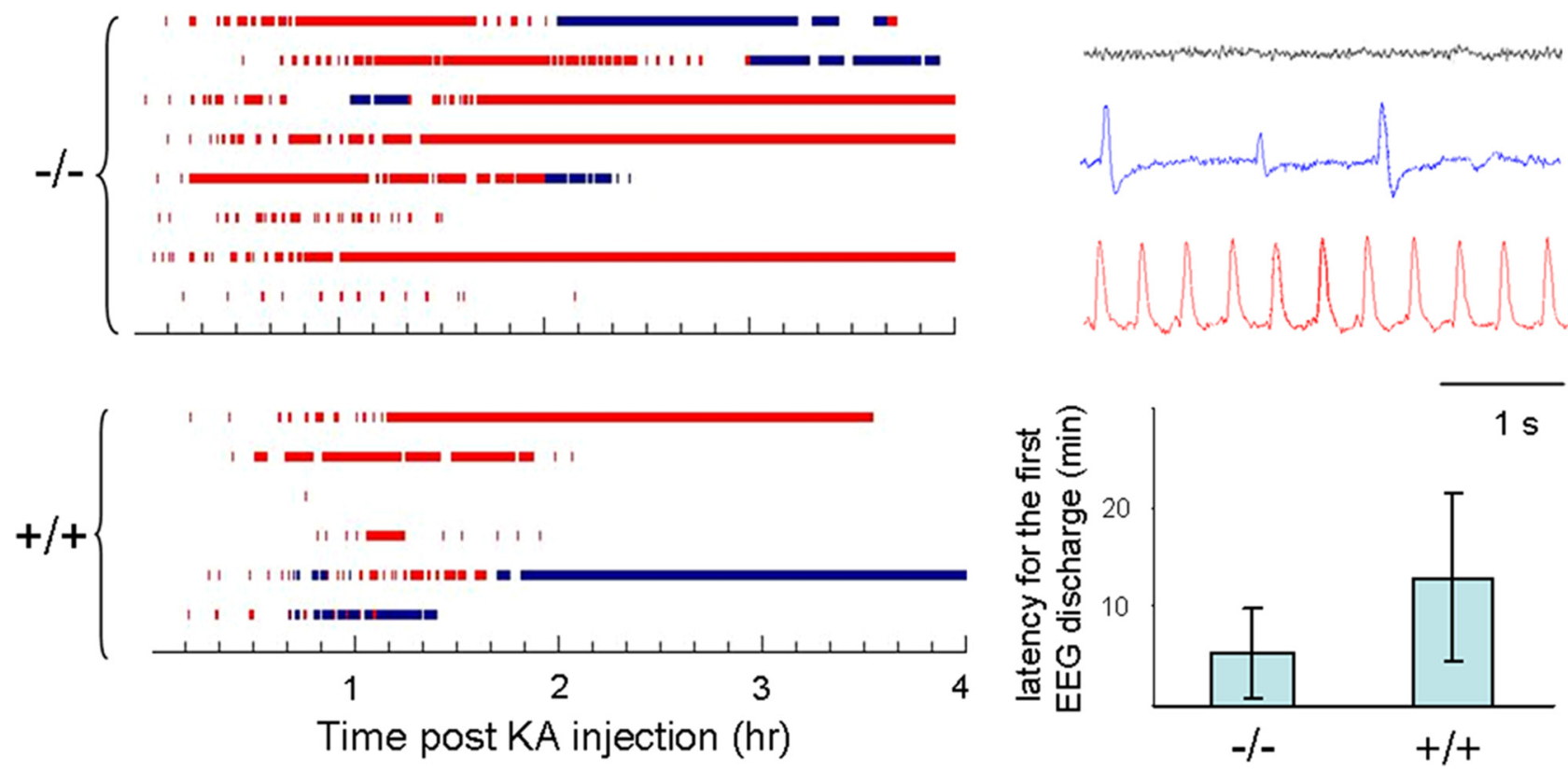

Figure 4. Higher seizure susceptibility in Zip-1,3-/- mouse brain. Summary data quantifying shorter latency to seizure onset and prolonged time spent in active seizure activity in mutant compared with controls injected with kainic acid. EEG seizure activity recorded from mice $(-/-n=8 ;+/+n=6)$ injected with KA (15 mg/kg). Before injection, Zip 1,3 mice show normal EEG patterns (right, gray sample trace) without any spontaneous cortical discharge activity similar to wild-type mice. After injection of KA, behavioral and electrographic seizure activity gradually developed over $30 \mathrm{~min}$ (blue and red sample traces). As shown at left bottom, the average latency for the initial onset of high-frequency EEG spiking was $11 \pm 9$ min in the $-/-$ group, which is less than one half that in the $+1+$ group $(26 \pm 17 \mathrm{~min}, p=0.05)$. Blue bar marks the time during which the EEG shows low-frequency $(<1 \mathrm{~Hz})$ aberrant synchronized spike discharges (blue sample trace) Red bar indicates the period of high-frequency synchronized EEG seizure discharges (red sample trace). By comparison of measures of latency to seizure onset and total time spent in seizure activity, Zip-1,3-/- mutants exhibit higher seizure susceptibility to the KA injection than their $+/+$ littermates.

In contrast, due to significant neuroprotection in the CA1 region, Zip-1,3-null mutants show damage largely confined to the CA3 region, with minor amounts of CA1 injury. Only 2 of 43 mutants showed evidence of isolated CA1 damage. The FJI for the CA3 area was positive in a similar proportion of Zip-1,3-null mice (26/43) compared with wild-type mice (26/41); however, the average FJI for CA3 in Zip 1,3-null mice was $29 \pm 27 \%(n=43)$ of its maximal value, which is significantly larger than the average value from the $+/+$ group $(19 \pm 23 \%, n=41, p=0.04)$, indicating more $\mathrm{CA} 3$ cell damage occurs in the $-/-$ group. Figure $3 C$ summarizes cell injury in each subregion of hippocampus.

\section{Zip-1,3-null mutants exhibit a lower seizure threshold to kainate}

The extent of hippocampal cell injury in experimental seizure models is strongly dependent on the severity of seizure activity. It is therefore possible that if developmental absence of $\mathrm{Zip}-1,3$ transporters lowered the underlying network excitability, weaker seizures, rather than reduced vulnerability to $\mathrm{Zn}$ entry, might explain the differences in cellular damage between the mutant and wild-type brains. To compare seizure severity between the genotypes, both behavioral and EEG seizure monitoring was continuously performed in mice from both groups during the period of KA exposure and accurately quantified by frequency analysis of the digitized EEG trace. Zip-1,3-null mutants display normal patterns of EEG activity during routine monitoring, but reveal a latent hyperexcitability upon administration of the glutamate agonist. Figure 4 shows EEG activity recorded from mice $(-1-, n=$ $8 ;+/+, n=6$ ) before and during the first $4 \mathrm{~h}$ after KA injection. Zip-1,3 mutants exhibited more synchronized high-frequency spike bursting (Fig. 4, marked in red) than wild-type mice, and 3 of 8 mutant mice showed essentially constant EEG seizure activity, lasting even beyond the $4 \mathrm{~h}$ experimental monitoring win-

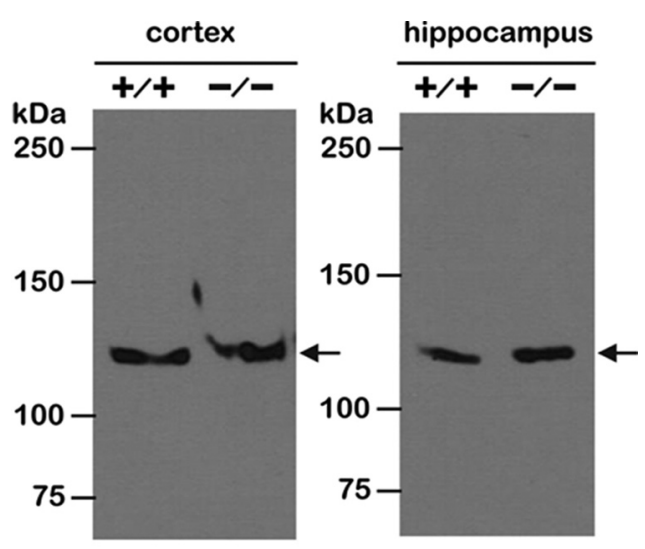

IB: NMDAR1 pAb

Figure 5. Unaltered NMDA receptor expression in Zip-1,3-/- mouse brain. NMDA receptor expression, a major alternative pathway for neurodegeneration following seizures was examined by Western blot with antibody against NMDAR1 subunit. Similar immunoreactive bands corresponding to the $120 \mathrm{kDa}$ NMDAR1 subunit were detected in cortex and hippocampus of $+/+$ and Zip-1,3- $-1-$ mouse brain, indicating unaltered global NMDA receptor expression in both cortex and hippocampus of the $-/-$ group.

dow. Among the six $+/+$ mice recorded for EEG activity, none displayed seizures beyond $4 \mathrm{~h}$, and the EEG seizure discharges were discontinuous. The average latency for the initial onset of high-frequency EEG spiking was $11 \pm 9 \min (n=8)$ in the $-/-$ group, which is less than one half that in the $+/+$ group $(26 \pm 17$ $\min , n=6, p=0.05)$. Seizure-induced lethality also differed between genotypes; $17 \%$ of the tested mutants died during a prolonged seizure, compared with only $4 \%$ in the $+/+$ group after injection of $15 \mathrm{mg} / \mathrm{kg} \mathrm{KA}$. At a higher dose $(30 \mathrm{mg} / \mathrm{kg})$, the difference in mortality was even greater (mortality rate was $66 \%$ 
and $22 \%$, respectively, for $-/-$ and $+/+$ mice). These results demonstrate that less seizure-induced neurodegeneration occurs in the CA1 area of Zip-1,3-/- mice even though they experience far more severe seizure activity after KA injection than the $+/+$ group.

During seizures, glutamate excitotoxicity, particularly mediated by NMDA receptors, also contributes to hippocampal pyramidal cell injury. If $Z i p-/-$ mice express a lower NMDA receptor density, then less neuronal cell damage in the $-1-$ group could be explained by a reduction in glutamate excitotoxicity. To evaluate this possibility, we compared the expression levels of NMDA receptors by Western blot between $+/+$ and $-/-$ mice. Figure 5 shows NMDA receptor expression in the cortex and hippocampus of $+/+$ and Zip-1,3 - /- mouse brains. Comparable immunoreactive bands corresponding to the $120 \mathrm{kDa}$ NMDAR1 subunit were detected in cortex and hippocampus, indicating unaltered global NMDA receptor expression in Zip-1,3 -/- mouse. This result also suggests that the increased seizure severity in Zip-1,3-/- brain is unlikely to be explained by secondary changes in NMDA receptor density.

\section{Less neuronal cell damage in Znt-3 -/-} mouse with KA injection

If postsynaptic $\mathrm{Zn}$ uptake contributes to pyramidal cell vulnerability, then reduction of extracellular $\mathrm{Zn}$ content during seizure activity might resemble the effect of $Z$ ip-1,3 transporter deletion. We examined early neurodegeneration in mice lacking synaptic $\mathrm{Zn}$ by injecting $\mathrm{Znt}$-3 $-/-$ mice with KA $(15 \mathrm{mg} / \mathrm{kg})$ in parallel studies. The same dose of KA, which usually evokes a full body convulsion in either the Zip-1,3-/- or +/+ group within the first $30 \mathrm{~min}$, only caused forelimb tremors in Znt-3 - / - mice within the first hour of the injection. After a second dose of 15 $\mathrm{mg} / \mathrm{Kg} \mathrm{KA}$ injection $1 \mathrm{~h}$ later, $Z n t-3-/-$ mice started to develop full body convulsions and were killed for Fluoro Jade staining $24 \mathrm{~h}$ later. Figure 6 summarizes the result for the $\mathrm{Znt}-3-\mathrm{I}-$ group. There is much less cell damage in both the CA 3 and CA1 areas after two injections of $15 \mathrm{mg} / \mathrm{kg} \mathrm{KA}$ compared with the $+/+$ group with one injection of $15 \mathrm{mg} / \mathrm{kg} \mathrm{KA}$. The overwhelming majority of mice did not show any cell damage in the CA1 area. Only 3 of $34 Z n t-3-/-$ mice fell in the upper 60 percentile of FJIs. The mean FJI in the CA1 area was $8 \pm 17 \%(n=34)$, which is significantly less than that in the $+/+\operatorname{group}(p=0.01)$. In the CA3 area, the average FJI was $9 \pm 12 \%(n=34)$, which is also significantly smaller than the average value from the $+/+$ group $(p=0.03$ ). As shown in Figure $6 B$, cell injury for both CA1 and CA3 remained in a smaller range compared with the $+/+$ group. Interestingly, EEG monitoring of Znt-3 - /- mice after only one injection of KA at $15 \mathrm{mg} / \mathrm{kg}$ showed more seizure activ-

D
B
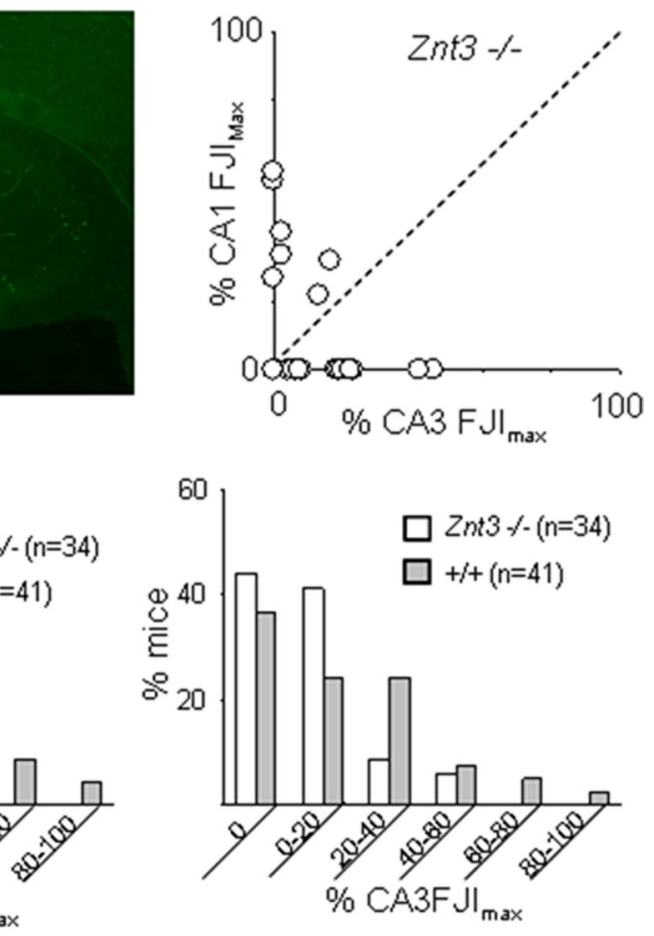

(n)

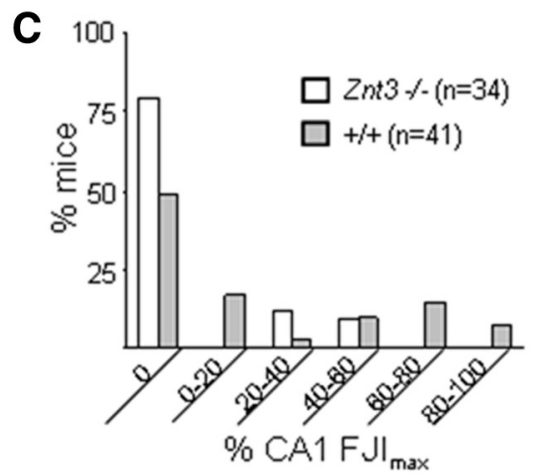

Figure 6. Less hippocampal cell damage in Znt3 - / - mouse brain following KA injection. A, A representative Fluoro-Jadewith $+/+$ group. $B$, In both CA1 and CA3 areas, cell injury is confined to a smaller region of the pyramidal cell layer. $C$, Direct comparison of cell damage between Znt-3 $-/-$ and $+/+$. About $75 \%$ of Znt3 $-/-$ mice tested showed no CA1 damage, $-1-$ group within both CA1 and CA3 regions. D, Quantitative plot of EEG seizure activity (red bar indicates high-frequency spiking $\mathrm{mg} / \mathrm{kg}$ ). Although the average latency for the initial onset of high-frequency EEG spiking was not significantly different from that in the $+/+$ group, on average the $-/$ - group displayed high EEG seizure activity for a much longer period.

ity than the $+/+$ group even though their behavioral seizure activity scores were low. Although their average latency for the initial onset of high-frequency EEG spiking was not significantly different from mice in the $+/+$ group, $Z n t-3-/-$ mice displayed longer, on average, seizure episodes of high-frequency EEG spiking. Our results indicate that reduction of $\mathrm{Zn}$ uptake, either by knockout of Zip-1,3 transporters or abolishment of synaptic Zn, can protect neurons from neurodegeneration following prolonged seizures.

\section{Discussion}

The ZIP Slc39a superfamily of metal transporters exists in all eukaryotes and imports $\mathrm{Zn}$ ions into the cytoplasm. Another family, Slc30a, is involved in Zn export (Kambe et al., 2004). ZIP proteins include eight transmembrane domains, the fourth of which contains fully conserved histidyl and glycyl residues in a 
putative amphipathic $\alpha$ helix. In mouse and humans, 14 genes encoding ZIP proteins have been identified with widely differing patterns of tissue expression; of these, 7 (Zip 1, 3, 4, 5, 10, 13, and 14) are variably expressed in brain (Dufner-Beattie et al., 2003; Belloni-Olivi et al., 2009; Emmetsberger et al., 2010) (Allen Brain Atlas). Although no specific antibodies for mammalian Zip proteins expressed in brain have been reported, our analysis is the first to identify the in vivo functional presence of these transporters in CNS neurons.

\section{Zip-1,3 transporters regulate $\mathrm{Zn}$ homeostasis in hippocampal neurons}

Loading high-affinity $\mathrm{Zn}$-sensitive fluorescent dye into patchclamped CA1 pyramidal cells bathed with D-APV and DNQX allowed us to demonstrate the passive uptake of $\mathrm{Zn}$ not mediated by voltage- or glutamate receptor-dependent channels in mouse brain slices. The relative reduction of passive $\mathrm{Zn}$ uptake in mutant neurons with targeted deletions of the plasma membrane $\mathrm{Zn}$ transporters $Z i p-1$ and $Z i p-3$ provides the first direct evidence for the involvement of members of the Zip transporter superfamily in the regulation of $\mathrm{Zn}$ homeostasis in hippocampal CA1 neurons. We found that Zip-1 and Zip-3 transporters account for $\sim 50 \%$ of the passive $\mathrm{Zn}$ uptake in these cells when extracellular $\mathrm{Zn}$ concentration reaches the low micrometer range $(10 \mu \mathrm{M})$, a concentration that is in line with their Michaelis-Menten kinetics. When assayed in cultured HEK293 cells, both Zip-1 and Zip-3 have an apparent $K_{\mathrm{m}}$ of $\sim 1.7 \mu \mathrm{M}$ (Dufner-Beattie et al., 2003). We used the Allen Brain Atlas to determine that two other genes of the Slc39A family, Zip-5 and Zip-10, show particularly strong hippocampal mRNA transcript expression and presumably contribute to the remaining passive influx. To localize the putative $Z i p-1$ and Zip-3 transporters in neuronal dendritic and somatic compartments, we tried three Zip-1 and Zip-3 antibodies made available by other laboratories; however, none proved to be specific in brain tissue. The exact cellular and compartmental location of $Z$ ip transporter proteins in central neurons remains to be further characterized.

\section{Slower Zn uptake reduces neurodegeneration in the CA1 area but not CA3 neurons}

The reduction of CA1 pyramidal cell injury in mouse mutants with targeted deletion of Zip-1 and Zip-3 also provides the first direct evidence that reduced postsynaptic Zn entry is neuroprotective in these cells. In addition to $\mathrm{Zn}$ transporters, $\mathrm{Zn}$ can also enter cells via $\mathrm{Ca}^{2+}$-permeable AMPA receptors lacking the GluR2 subunit. CA1 pyramidal cells in the mature hippocampus abundantly express AMPA receptors containing GluR2 subunit (Wenthold et al., 1996). Under pathological situations such as seizures and ischemia, these AMPA receptors can undergo subunit reorganization (Grooms et al., 2000; Noh et al., 2005; Kwak and Weiss, 2006; Liu and Zukin, 2007), and the resulting GluR2deficient AMPA receptors are permeable to both $\mathrm{Ca}^{2+}$ and $\mathrm{Zn}^{2+}$. While these receptors contribute to cell death after ischemia (Yin et al., 2002), it is not clear how much they contribute to Zn entry. Despite their activation during KA-induced seizures and potential Zn entry through NMDA receptor and voltage-gated $\mathrm{Ca}$ channels at synaptic sites, our results indicate that tonic $\mathrm{Zn}$ uptake by $\mathrm{Zn}$ transporters contributes to a significant additional fraction of early hippocampal cell injury. Interestingly, the pattern of cell damage due to loss of Zip-1,3 was not uniform in the hippocampus. As shown by EEG recordings and the increased mortality rate following KA injection, even though $Z i p-1,3$-null mutants were more susceptible to KA-induced seizures, they showed less neuronal cell injury in the CA1 pyramidal region. In contrast, in the CA3 area more pyramidal cell injury was observed in mutants than wild-type control mice. Several factors may contribute to the disparity in different hippocampal regions, including a difference in activity-induced release of $\mathrm{Zn}$ at the two sites. Due to the extensive innervation by $Z n$-rich mossy fibers and severer seizure activity, massive $\mathrm{Zn}$ release and $\mathrm{Zn}$ uptake in CA3 of the $-/-$ group during kainate seizures may surpass that seen in CA1 even though $Z$ ip-1,3 transporters are not present in those CA 3 cells. Less cell death in CA1 but more in CA3 following acute brain injury has also been reported in mice deficient in metallothionein, a major intracellular $\mathrm{Zn}$ binding protein (Lee et al., 2003). Intrinsic activity-dependent mechanisms other than $\mathrm{Zn}$ release, entry and synaptic connectivity may also contribute to region-specific variation in excitability and neurodegeneration levels, as confirmed by our Znt-3 - / - experiments that showed minor cell injury after seizures even in a hippocampus devoid of synaptic Zn.

\section{Synaptic Zn contributes to neurodegeneration of pyramidal cells in the hippocampus}

In the present study, we also show that lack of synaptic Zn leads to less pyramidal cell neurodegeneration in both CA1 and CA3 areas following KA injection of $Z n t-3-/-$ mice. A previous study using the same $Z n t-3-/-$ mutant mice reported that although the CA 3 area exhibited less cell damage than $+/+$ wild-type mice, little difference was found in the CA1 area (Lee et al., 2000). Our results generally confirm these findings, and several factors may contribute to the disparity that CA1 neurons were severely damaged in their study, while the same region was relatively spared in ours. First, a higher KA dose was given to induce status epilepticus in their study then used here. Therefore, more severe seizure activity and a higher KA peak concentration in the brain, along with the loss of $\mathrm{Zn}$-mediated inhibition of NMDA receptors in Znt-3 -/- mice might amplify overall glutamate excitotoxicity in that study. Consequently, the contribution of $\mathrm{Zn}$ entrymediated cell injury may be masked by the greater amount of NMDA receptor mediated, Ca-induced neurodegeneration in the CA1 area, a region well known for its vulnerability to glutamate excitotoxicity. Second, prolonged seizures were a selection criterion for analysis in their study, whereas we studied mice following more moderate seizure activity. To control for the relatively high interanimal variation in cell degeneration patterns found in convulsant models, we investigated cell injury in a very large experimental cohort. Extensive CA1 pyramidal cell damage, as identified in the top 60th percentile, could still be detected in mice lacking synaptic $\mathrm{Zn}$ but with a much lower incidence $(3 / 34)$ compared with their wild-type littermates (13/41). The results obtained in both studies of $Z n t-3-/-$ mice are consistent with our demonstration that entry of $\mathrm{Zn}$ into postsynaptic cells contributes to neurodegeneration in the early stages of brain injury.

\section{References}

Bancila V, Nikonenko I, Dunant Y, Bloc A (2004) Zinc inhibits glutamate release via activation of pre-synaptic $\mathrm{K}$ channels and reduces ischaemic damage in rat hippocampus. J Neurochem 90:1243-1250.

Belloni-Olivi L, Marshall C, Laal B, Andrews GK, Bressler J (2009) Localization of zip1 and zip4 mRNA in the adult rat brain. J Neurosci Res 87:3221-3230.

Besancon E, Guo S, Lok J, Tymianski M, Lo EH (2008) Beyond NMDA and AMPA glutamate receptors: emerging mechanisms for ionic imbalance and cell death in stroke. Trends Pharmacol Sci 29:268-275.

Cataldi M, Lariccia V, Marzaioli V, Cavaccini A, Curia G, Viggiano D, Canzoniero LM, di Renzo G, Avoli M, Annunziato L (2007) Zn(2+) slows 
down $\mathrm{Ca}(\mathrm{V}) 3.3$ gating kinetics: implications for thalamocortical activity. J Neurophysiol 98:2274-2284.

Colvin RA, Fontaine CP, Laskowski M, Thomas D (2003) Zn2 + transporters and Zn2+ homeostasis in neurons. Eur J Pharmacol 479:171-185.

Côté A, Chiasson M, Peralta MR 3rd, Lafortune K, Pellegrini L, Tóth K (2005) Cell type-specific action of seizure-induced intracellular zinc accumulation in the rat hippocampus. J Physiol 566:821-837.

Domínguez MI, Blasco-Ibáñez JM, Crespo C, Marqués-Marí AI, MartínezGuijarro FJ (2003) Zinc chelation during nonlesioning overexcitation results in neuronal death in the mouse hippocampus. Neuroscience 116:791-806.

Domínguez MI, Blasco-Ibáñez JM, Crespo C, Nacher J, Marqués-Marí AI, Martínez-Guijarro FJ (2006) Neural overexcitation and implication of NMDA and AMPA receptors in a mouse model of temporal lobe epilepsy implying zinc chelation. Epilepsia 47:887-899.

Dufner-Beattie J, Langmade SJ, Wang F, Eide D, Andrews GK (2003) Structure, function, and regulation of a subfamily of mouse zinc transporter genes. J Biol Chem 278:50142-50150.

Dufner-Beattie J, Huang ZL, Geiser J, Xu W, Andrews GK (2006) Mouse ZIP1 and ZIP3 genes together are essential for adaptation to dietary zinc deficiency during pregnancy. Genesis 44:239-251.

Emmetsberger J, Mirrione MM, Zhou C, Fernandez-Monreal M, Siddiq MM, Ji K, Tsirka SE (2010) Tissue plasminogen activator alters intracellular sequestration of zinc through interaction with the transporter ZIP4. J Neurosci 30:6538-6547.

Frederickson CJ, Koh JY, Bush AI (2005) The neurobiology of zinc in health and disease. Nat Rev Neurosci 6:449-462.

Frederickson CJ, Giblin LJ 3rd, Balaji RV, Rengarajan B, Masalha R, Frederickson CJ, Zeng Y, Lopez EV, Koh JY, Chorin U, Besser L, Hershfinkel M, Li Y, Thompson RB, Krezel A (2006) Synaptic release of zinc from brain slices: factors governing release, imaging, and accurate calculation of concentration. J Neurosci Methods 154:19-29.

Freund WD, Reddig S (1994) AMPA/Zn(2+)-induced neurotoxicity in rat primary cortical cultures: involvement of L-type calcium channels. Brain Res 654:257-264.

Grooms SY, Opitz T, Bennett MV, Zukin RS (2000), Status epilepticus decreases glutamate receptor $2 \mathrm{mRNA}$ and protein expression in hippocampal pyramidal cells before neuronal death. Proc Natl Acad Sci U S A 97:3631-3636.

Hershfinkel M, Kandler K, Knoch ME, Dagan-Rabin M, Aras MA, Abramovitch-Dahan C, Sekler I, Aizenman E (2009) Intracellular zinc inhibits KCC2 transporter activity. Nat Neurosci 12:725-727.

Hirzel K, Müller U, Latal AT, Hülsmann S, Grudzinska J, Seeliger MW, Betz H, Laube B (2006) Hyperekplexia phenotype of glycine receptor alpha1 subunit mutant mice identifies $\mathrm{Zn}(2+)$ as an essential endogenous modulator of glycinergic neurotransmission. Neuron 52:679-690.

Kambe T, Yamaguchi-Iwai Y, Sasaki R, Nagao M (2004) Overview of mammalian zinc transporters. Cell Mol Life Sci 61:49-68.

Koh JY, Suh SW, Gwag BJ, He YY, Hsu CY, Choi DW (1996) The role of zinc in selective neuronal death after transient global cerebral ischemia. Science 272:1013-1016.

Kwak S, Weiss JH (2006) Calcium-permeable AMPA channels in neurodegenerative disease and ischemia. Curr Opin Neurobiol 16:281-287.
Lavoie N, Peralta MR 3rd, Chiasson M, Lafortune K, Pellegrini L, Seress L, Tóth K (2007) Extracellular chelation of zinc does not affect hippocampal excitability and seizure-induced cell death in rats. J Physiol 578: 275-289.

Lee JY, Cole TB, Palmiter RD, Koh JY (2000) Accumulation of zinc in degenerating hippocampal neurons of ZnT3-null mice after seizures: evidence against synaptic vesicle origin. J Neurosci 20:RC79.

Lee JY, Kim JH, Palmiter RD, Koh JY (2003) Zinc released from metallothionein-iii may contribute to hippocampal CA1 and thalamic neuronal death following acute brain injury. Exp Neurol 184:337-347.

Liu SJ, Zukin RS (2007) Ca2+-permeable AMPA receptors in synaptic plasticity and neuronal death. Trends Neurosci 30:126-134.

Molnár P, Nadler JV (2001) Synaptically-released zinc inhibits N-methyl$\mathrm{D}$-aspartate receptor activation at recurrent mossy fiber synapses. Brain Res 910:205-207.

Noh KM, Yokota H, Mashiko T, Castillo PE, Zukin RS, Bennett MV (2005) Blockade of calcium-permeable AMPA receptors protects hippocampal neurons against global ischemia-induced death. Proc Natl Acad Sci U S A 102:12230-12235.

Qian J, Noebels JL (2005) Visualization of transmitter release with zinc fluorescence detection at the mouse hippocampal mossy fibre synapse. J Physiol 566:747-758.

Sensi SL, Canzoniero LM, Yu SP, Ying HS, Koh JY, Kerchner GA, Choi DW (1997) Measurement of intracellular free zinc in living cortical neurons: routes of entry. J Neurosci 17:9554-9564.

Sensi SL, Yin HZ, Carriedo SG, Rao SS, Weiss JH (1999) Preferential Zn2+ influx through $\mathrm{Ca} 2+$-permeable AMPA/kainate channels triggers prolonged mitochondrial superoxide production. Proc Natl Acad Sci U S A 96:2414-2419.

Takeda A, Tamano H, Nagayoshi A, Yamada K, Oku N (2005) Increase in hippocampal cell death after treatment with kainate in zinc deficiency. Neurochem Int 47:539-544.

Traboulsie A, Chemin J, Chevalier M, Quignard JF, Nargeot J, Lory P (2007) Subunit-specific modulation of T-type calcium channels by zinc. J Physiol 578:159-171.

Villmann C, Becker CM (2007) On the hypes and falls in neuroprotection: targeting the NMDA receptor. Neuroscientist 13:594-615.

Vogt K, Mellor J, Tong G, Nicoll R (2000) The actions of synaptically released zinc at hippocampal mossy fiber synapses. Neuron 26:187-196.

Weiss JH, Sensi SL, Koh JY (2000) Zn(2+): a novel ionic mediator of neural injury in brain disease. Trends Pharmacol Sci 21:395-401.

Wenthold RJ, Petralia RS, Blahos J II, Niedzielski AS (1996) Evidence for multiple AMPA receptor complexes in hippocampal CA1/CA2 neurons. J Neurosci 16:1982-1989.

Westbrook GL, Mayer ML (1987) Micromolar concentrations of Zn2+ antagonize NMDA and GABA responses of hippocampal neurons. Nature 328:640-643.

Yin HZ, Sensi SL, Ogoshi F, Weiss JH (2002) Blockade of Ca2+-permeable AMPA/kainate channels decreases oxygen-glucose deprivation-induced $\mathrm{Zn} 2+$ accumulation and neuronal loss in hippocampal pyramidal neurons. J Neurosci 22:1273-1279. 